\title{
34th Kinki Neuropathology Conference
}

\author{
(Prof. Scheithauer's slide session)
}

\author{
14, April 1992, Daiichi Building, Osaka
}

Chairmen: Yasuaki Nakashima (Kyoto University Hospital, Surgical pathology)

Keiji Kawamoto (Kansai Medical University, Neurosurgery)

\section{Special Lectur \\ "Newly Propoced WHO Histological Typing of the Central Nervous System"}

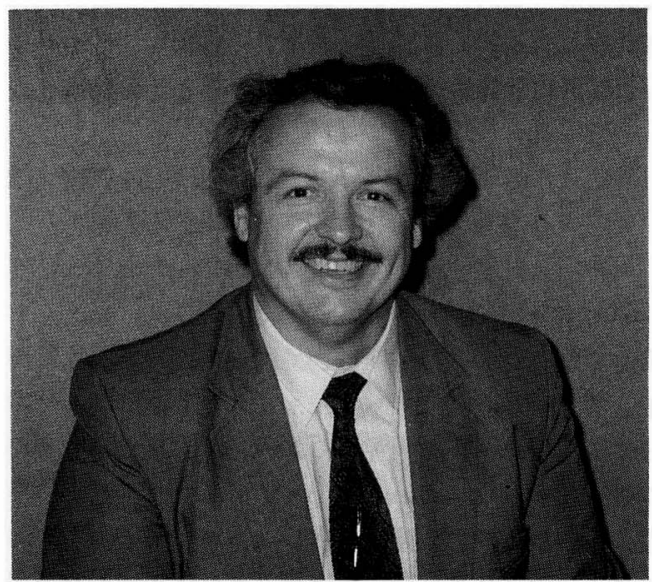

Prof. Bernd Walter Scheithauer

1946

1969

1973

1974 1978

$1979 \sim 1980$

$1980 \sim 1984$

$1980 \sim 1987$

1987
Born in Germany.

B. A. California State University.

M. D. Loma Linda University, CA.

Resident, Stanford University, CA.

Instructor, Pathology,

Mayo Medical School.

Assistant Professor.

Associate Professor.

Professor, Pathology,

Mayo Medical School.

\section{Malignant lymphoma or multiple sclerosis?}

Kyoto Minami Hospital, Departments of Neurosurgery, Neurology*, Pathology**

N. Kochi, T. Ametani, M. Makino*, K. Higuchi**

Radiological and neuropathological findings of a case with two possible but different diagnoses, malignant lymphoma (ML) or multiple sclerosis (MS), was presented. At first a $40 \mathrm{y}-\mathrm{o}$ housewife developed typical symptoms of intracranial tumor with raised intracranial pressure and spaceoccupying mass in the $\mathrm{rt}$ frontal lobe on CT and MRI. But surgical specimens (1991. 4. 25.) disclosed only inflammatory appearance, consisting of proliferating astroglia interspersed by numerous foamy macrophages, and perivascular lymphocyte cuffings. The feature was similar to bioptical findings of multiple sclerosis mimicking primary brain tumor described by Hunter et al. in 1987. Prior to operation, she had received glucocorticoid therapy (12mg q. d. dexamethasone for 6 days), which led remarkable improvement of neurological state. So the clinical side insisted on the diagnosis of malignant lymphoma obscured by glucocorticoid therapy because of (1) the patient's past history of ML in the axillary lymph node 9 years previously, (2) relative rarity of MS in Japan, and (3) glucocorticoid administration prior to surgery. Rapid regression 


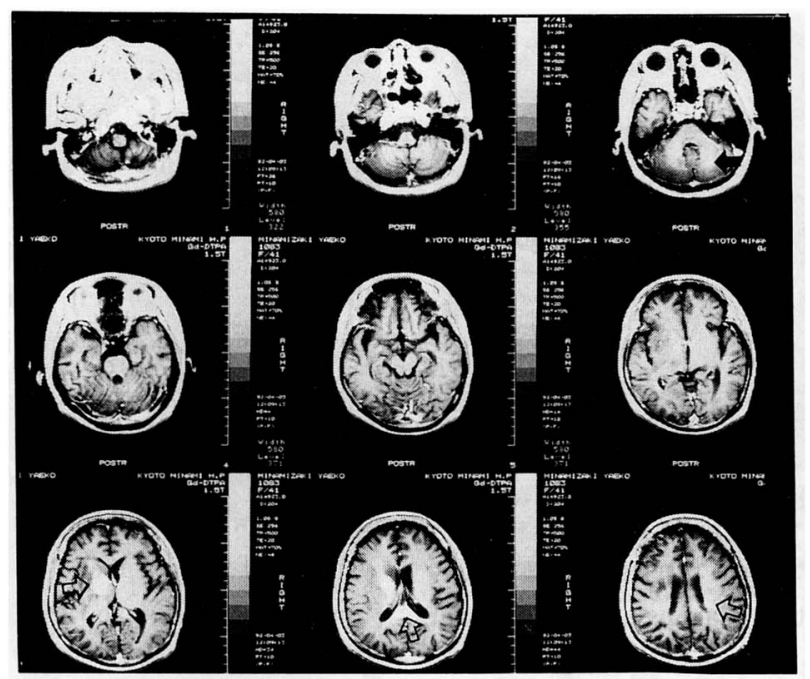

Fig. 1

of ML was well known as "ghost tumor", described by Vaquero et al. in 1984. Radiation and following COP chemotherapy were done, although repeated warnings were given by the pathological side with a kind advice of Prof. Scheithauer (demyelinating disease: 1991. 6. 12.). She was doing well till the present recurrence (disorientation as to time, short concentration span, untidy manner, ataxia in the lt. extremities and unsteady gait with the tendency to fall toward the left. Recent CT and MRI showed multifocal enhanced foci in rather selective involvement of white matter in rt. basal ganglia, lt. periventricular white matter, splenium of corpus callosum and both middle cerebellar peduncles (Fig. 1). For sure diagnosis, CT-guided stereotactic biopsy was performed on 9. Apr. 1992.

Histologically there were multiple infiltration foci of large lymphoid tumor cells, resembling the tumor cells in the lymph node specimen that had been extirpated in 1982. Many mitotic figures were seen (Fig. 2). The immunostaining showed abundant expression of the B-cell marker (L-26) in the cell membrane and the cytoplasm of many tumor cells (Fig. 3). Tumor cells were stained also for leukocyte-commonantigen (Fig. 4), but never developed T-cell markers (MT-1 or UCHL).

Comments: Dr. Scheithauer pointed out that this case was a typical pitfall on the diagnosis of malig-

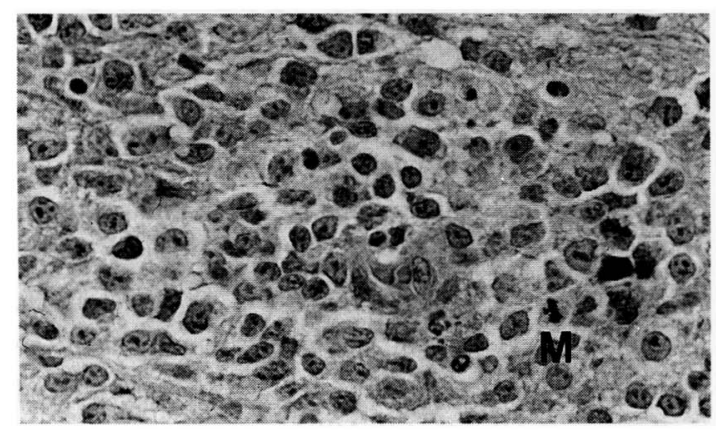

Fig. 2

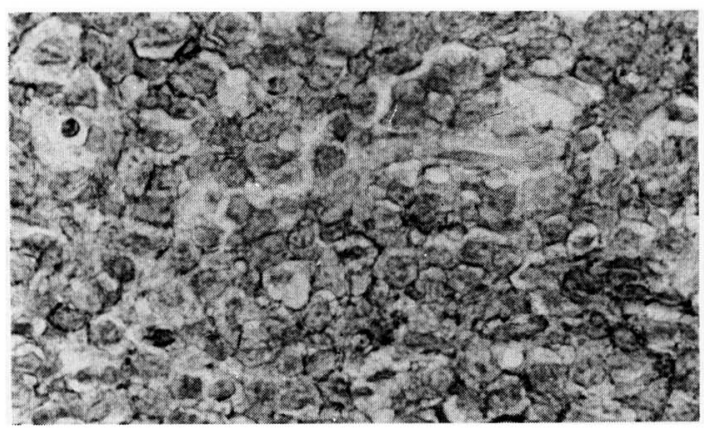

Fig. 3

nant lymphoma. He was not so informed of the clinical data, especially about preoperative administration of high dose of dexamethasone.

The instructive fact was that first we should not use glucocorticoids prior to biopsy on suspected 


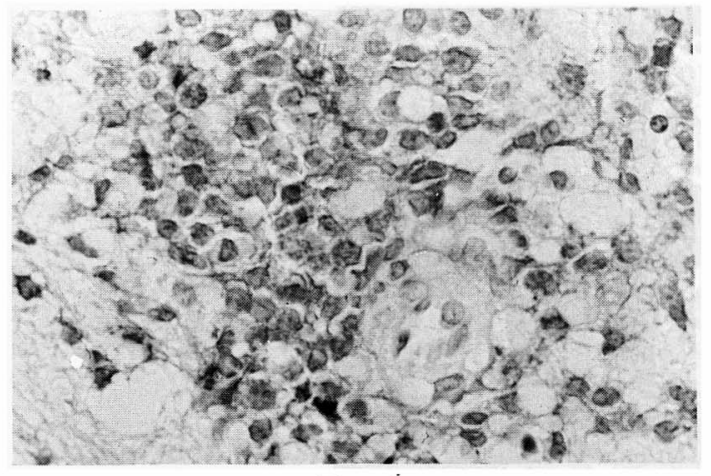

Fig. 4

malignant lymphoma. Second stereotactic biopsy was very useful for the diagnosis of malignant lymphoma as shown by Dr. Scheithauer et al.

\section{References:}

- Gepper M, Ostertag CB, Seitz G, Kiessling M: Glucocorticoid therapy obscures the diagnosis of cerebral lymphoma. Acta Neuropathol 80: 629634,1990

- Hunter SB, Ballinger WE, Rubin JJ: Multiple sclerosis mimiching primary brain tumor. Arch Pathol Lab Med 111: 464-468, 1987

- Makino M, Takezawa N, Kochi N, Ametani T, Higuchi K: A case of malignant lymphoma in the brain (ghost tumor ?). Minamibyouin Med J (Iapanese) 10: 52-58, 1991

- O’Neill BP, Kelly PJ, Earle JD, Scheithauer B, Banks PM: Computer-assisted stereotaxic biopsy for the diagnosis of primary central nervous system lymphoma. Neurol 37: 1160-1164, 1987

- Vaquero J, Martinez R, Rossi E, Lopez R: Primary cerebral lymphoma: the "ghost tumor". J Neurosurg 60: 174-176, 1984

\section{Intracranial Plasmacytoma}

Department of Neurosurgery, Yodogawa Christian Hospital.

Shuji Kamikawa, M. D., Haruo Yamashita, M. D., and Seiya Shirakata, M. D.

\section{[Case]}

A 48-year-old man was admitted to our hos- pital with slowly progressive gait disturbance. On admission, neurological examination revealed mild right hemiparesis. Plain $\mathrm{X}$-ray films of the skull were normal. The CT scan showed an irregular enhanced lesion with severe edema in the left frontal lobe. The MR image revealed the lesion located at the medial part of left frontal cortex with spreading into interhemispheric fissure and brain surface diffusely.

At operation, the elastic hard greyish tumor like lesion was found in the moderately edematous left frontal cortex. The dura matter, Falx cerebri, and arachnoid membrane were thickened at this portion. Subtotal removal of tumor was performed.

\section{[Pathology]}

Histologically, the lesion consists of fibrovascular tissue with heavy infiltrates of plasma cells. Scattered lymphocytes (B-cell) were also present. Whorl formations and psammomatous bodies were absent. The PAS and Grocott stains revealed no organisms.

\section{[Comments]}

With marked plasma cell infiltrate, plasmacyto ma was considered. In this case, immunohistochemical analysis demonstrated the polyclonal nature of the plasma cell proliferation. From this point of view, it may be inflammatory proliferation. (e. g. plasma cell granuloma)

\section{Does true polar spongioblastoma exist?}

Department of Neurosurgery, Hyogo College of Medicine.

T. Morimura, K. Kaba, T. Matsumoto, E. Tani

This $45 \mathrm{y}-\mathrm{o}$, right-handed housewife (T. O., 268692) was admitted to our ward complaining of unconscious attack of late onset. On admission neurological exam. revealed no abnormality. Her fanily and past histories were unremarkable. A CT scan showed an isodensity round mass with slight edema in left frontal lobe. (Fig. 1) The tumor had homogenous contrast enhancement. Angiography disclosed an avascular mass with round shift of ACA to the left. At operation, grayish, soft tumor 


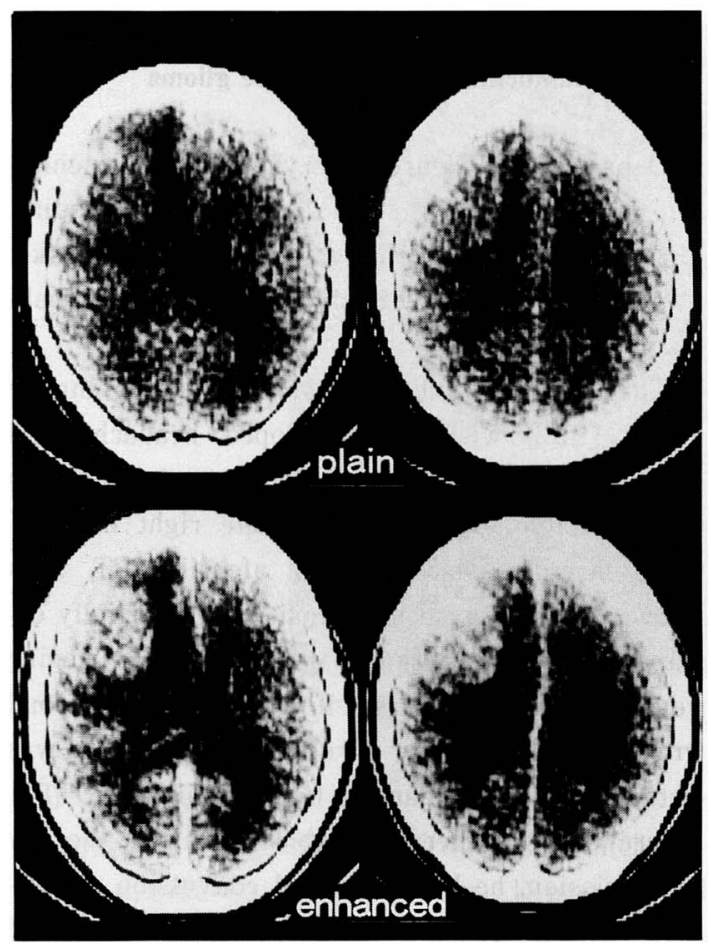

Fig. 1 This $45 \mathrm{y}-0$ female had two episode of unconscious attack three years before admission. On CT scan, round, well circumscribed, isodensity mass with slight edema was recognized (upper) and homogenously enhanced (lower).

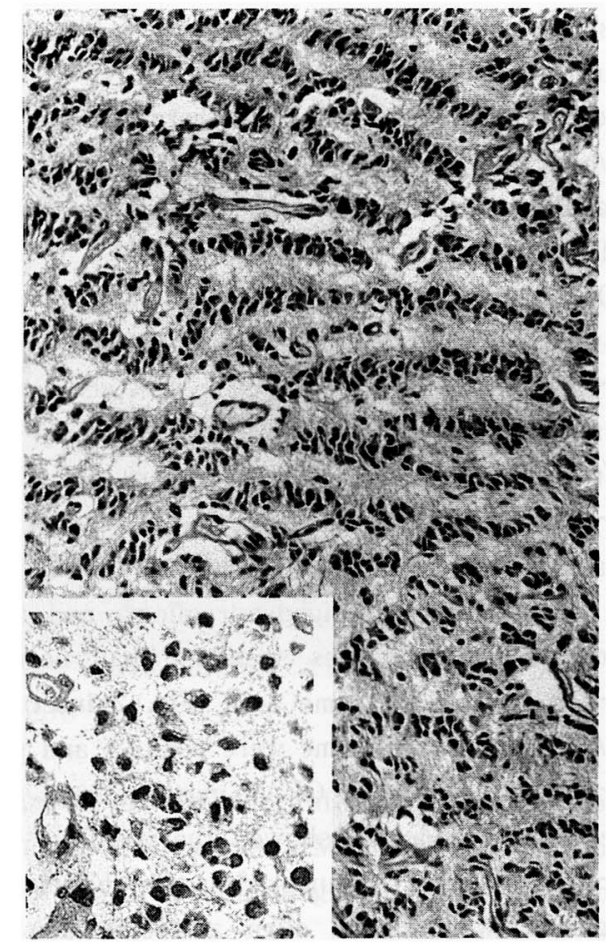

Fig. 2 The tumor specimen shows highly distinctive cytoarchitecture like "terraced field". Cells are arranged in parallel fashion of $2-3$ rows between acellular zone. In some areas the feature of oligodendroglioma such as "fried egg" was recognized among vascular connective tissue stroma. (inset) $\mathrm{H} \& \mathrm{E} \times 100 \& 200$

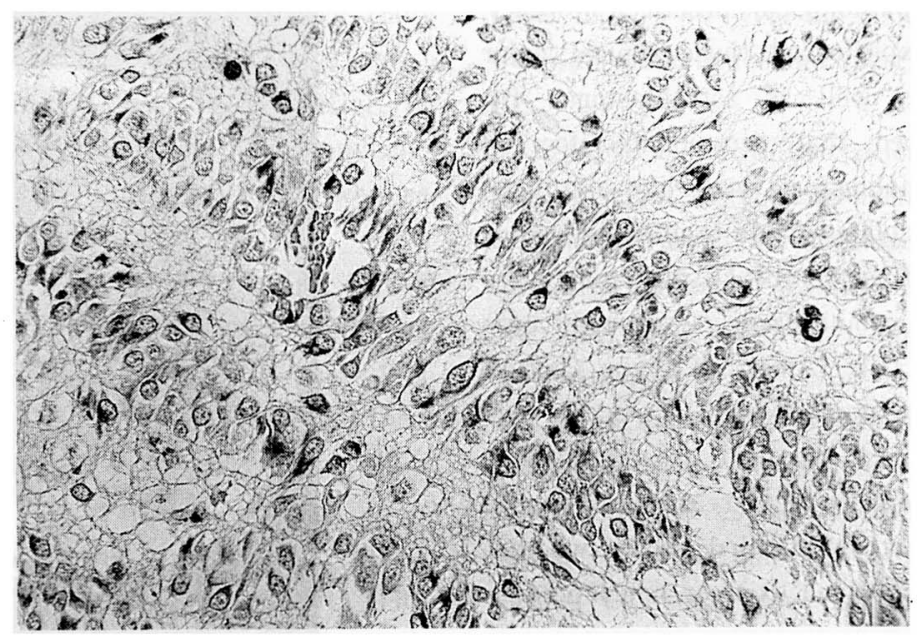

Fig. 3 There are some strong GFAP reactivity in tumor cells. GFAP stain $\times 200$ 
was well-demarcated and removed en bloc. Postoperative course was uneventful and the patient received irradiation and chemotherapy. Now over 7 years after operation, she is still living, somewhat obtunded, probably due to radiation therapy and tumor-free on follow-up CT scans.

On histological exam. the tumor specimen showed highly distinctive arrangement of cells like "terraced field". (Fig. 2) The cells were arranged in parallel fashion of 2-3 rows, forming fairly compact bands or palisades. The tumor cell groups were separated by a delicate and regularly arranged vascular connective tisue stroma. Typical cell arrangement of oligodendroglioma and minimum anaplastic changes such as small necrosis were recognized in some area (Fig. 2 inset). On GFAP immunostain, some were positive and some were negative. (Fig. 3) GFAP-positive cells were intermingled and this showed that positive cells were not reactive but tumor cells. Our histological diagnosis was mixed oligo-astrocytoma. Prof. Scheithauer diagnosed this fascinating tumor as polar spongioblastoma and said that the tumor should not be called a mixed lesion but polar spongioblastoma with focal oligodendroglial differentiation. The true polar spongioblastoma as originally defined by Russel and Cairns in 1947 seen in the preparations of the cerebrum in 16-week-old embryo. On neuropathological practice, however, we have rarely experienced those typical polar spongioblastoma as well as astroblastoma. Dr. Rubinstein described that occasionally foci of "pure" polar spongioblastoma may, like astroblastic foci, be met in isolated fields in glioblastoma or dedifferentiating diffuse cerebral astrocytomas. We performed $\mathrm{A}_{2} \mathrm{~B}_{5}$ immunostain. $\mathrm{A}_{2} \mathrm{~B}_{5} \mathrm{mAb}$ was purchased from ATCC (American Type Culture Collection) and this stains type 2 astrocytes and oligodendrocyte-type 2 astrocyte progenitor cells. We found that there were weak reaction as seen in mixed gliomas. Further detailed study whether true polar spongioblastoma exist or not should be made.

\section{Acute desseminated encephalomyelitis (ADEM) mimicking a temporal lobe glioma}

Department of Neurosurgery, Osaka Koseinenkin Hospital Hiroshi Hasegawa, Kousuke Iwaisako, Kentaro Koshino, Yasushi Kobayashi

This 19-year-old male was well until January 14th, 1992, when he developed headache and visual field defect. Neurological examination was unremarkable except for complete right homonymous hemianopsia. He was afebrile. CT scan showed left temporal low density area partially enhanced by contrast material (Fig. 1). MR imaging revealed left temporal swelling and loss of normal gray and white matter structures. The lesion was heterogeneously enhanced by Gadolinium-DTPA. There was no other detectable lesion. On the day of admission, he had a general convulsion. Operative findings (01-29-92): Corticotomy revealed grayish elastic hard tumor in the left temporal lobe. Frozen section of the biopsy specimen was reported as a low grade astrocytoma. The temporal lobectomy was performed.

Postoperative course: His immediate postoperative course was uneventful but a week later, he

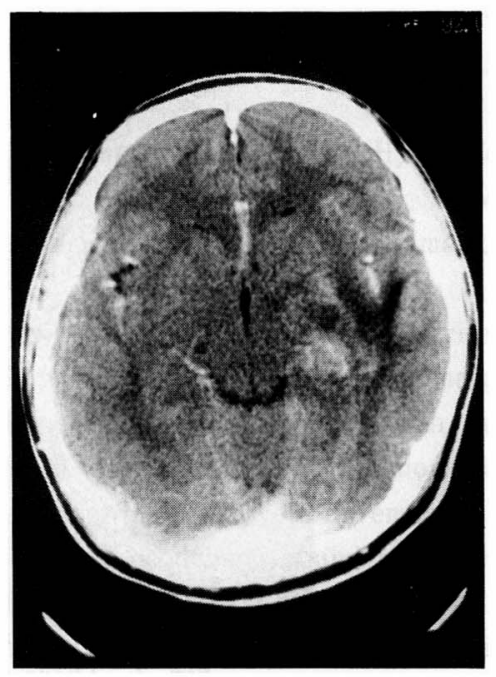

Fig. 1 Enhanced CT 


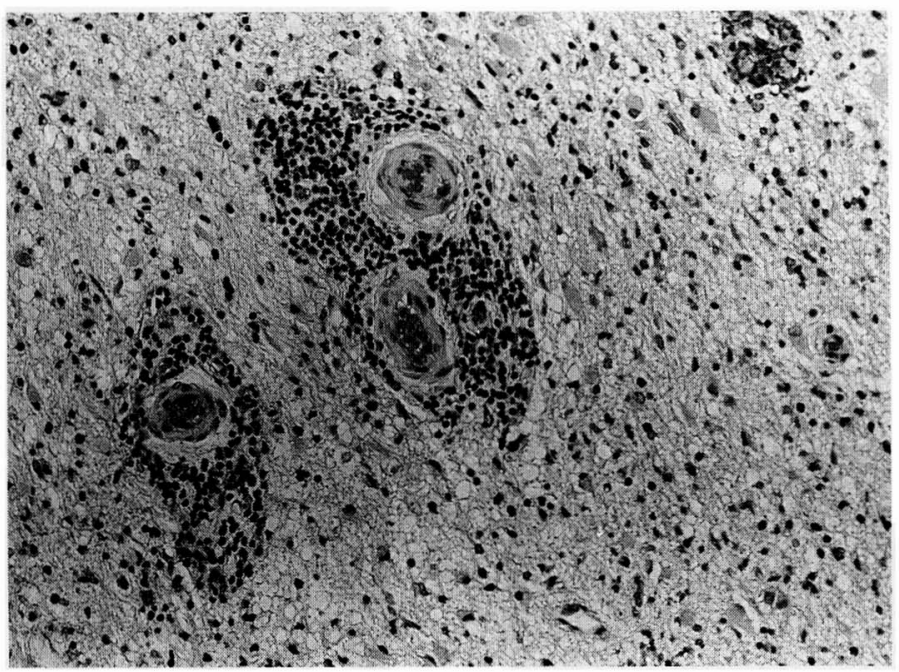

Fig. 2 H \& E $\times 200$

had fever and persistent hiccup, followed by obtundation and respiratory distress which required intubation and respirator. Repeated MRI showed extensive lesions in the midbrain, pons, medulla and cerebellum. Lumbar CSF contained 388 WBCs (Poly 60\%, Lymph 40\%), total protein $99 \mathrm{mg} / \mathrm{dl}$, and glucose $55 \mathrm{mg} / \mathrm{dl}$. CSF cytology revealed moderate number of atypical immature lymphocytes. Both CSF oligoclonal band and myelin basic protein were negative. Extensive virological studies, including EB, HS, HZ, rubella, and mumps except for rubeola (1:16) were all negative. Daily Aciclovir injection did not alter the clinical course but steroid pulse therapy was very effective. He recovered well and his right homonymous hemianopsia improved. Repeated MRI showed resolution of the brain stem lesions.

Histological findings: The lesion contains foci of necrosis and hemorrhage with prominent perivascular lymphocytic cuffings (Fig. 2). These lymphocytes are mixture of $\mathrm{T}-$ and $\mathrm{B}$-cells and there are areas of reactive astrocytosis and demyelination.

Comments: With the advent of MR imaging, there are increasing case reports of ADEM but its histological findings are rarely reported. ADEM usually manifests itself as multiple CNS lesions but when initial radiological studies reveal a single lesion, diagnosis of ADEM is difficult without a biopsy. Although it is rare, ADEM should be added to the list of the differential diagnosis for intraparechymal lesion.

\section{A recurrent case of hemangioblastoma}

Dept. of Neurosurgery, Kansai Medical University Keiji Kawamoto, Harufumi Kasai, Yasuhiro Kaji, Takushi Nishimura

Case: T. A., a 34-year old male

CC: Gait disturbance and speech disturbance

PI:

On April 9, 1986, the patient underwent resection of right cerebellar hemangioblastoma at a medical university. He developed right hearing disturbance, cerebellar ataxia, and right facial palsy after operation, but these symptoms were considerably improved.

Around the middle of January 1992 his speech became disconnective and his gait was rather unsteady. Headahces also became worse. He consulted our clinic in February 1992. Since performed CT scans revealed abnormality, he was hospitalized in our hospital on March 9, 1992.

\section{Neurological examinations:}

There were ataxic gait, speech disturbance, right 
hearing disturbance, and right facial palsy.

CT and MRI findings:

There was a solid mass in the center of the right cerebellopontien angle region with a cyst in the medial part, which compressed the brain stem. Contrast enhancement clearly revealed a solid mass. (Fig. 1)

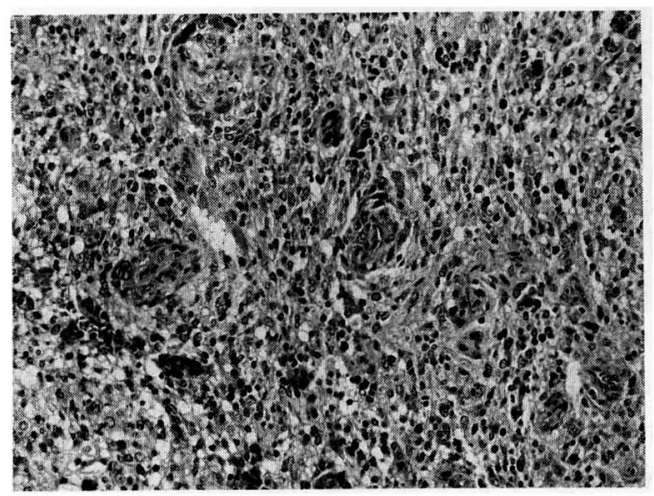

Fig. 1

\section{Operation:}

On March 19, 1992 subtotal removal of the right cerebellar tumor was performed through the right suboccipital craniectomy.

A soft hemorrhagic solid tumor and yellowish cyst existing in the right cerebellopontine angle to inside the cerebellum were resected. The exterior part of the tumor was rather soft with relatively clear margin.

\section{Histology:}

Some parts of the tumor show a typical hemangioblastoma. Another parts of the tumor show

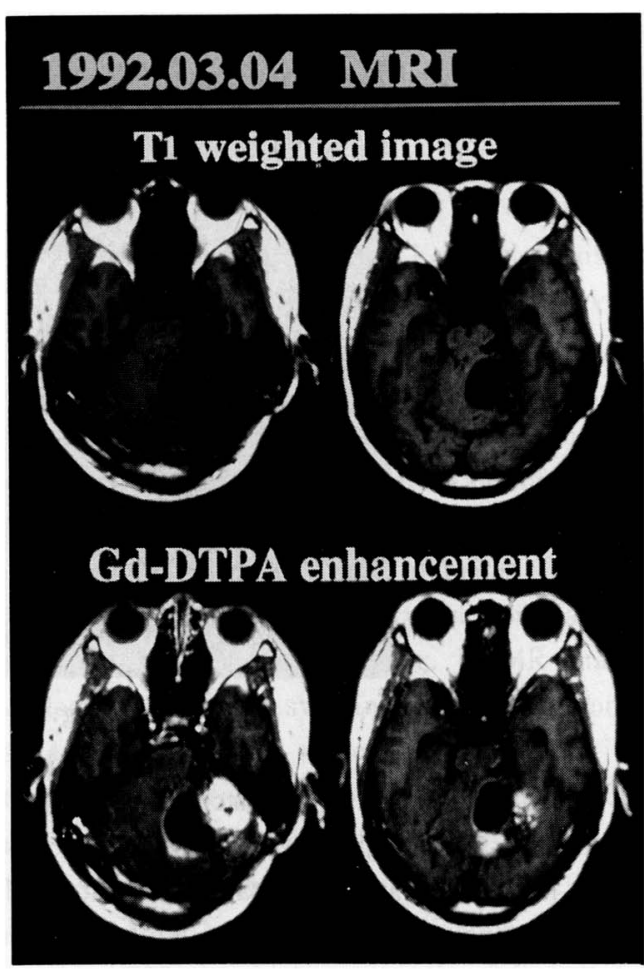

Fig. 2

slender processes with positive GFAP staining and many endotherial proliferation. (Fig. 2)

\section{Diagnostic problem:}

Recurrence of hemangioblastoma with gliosis or glioma.

\section{Comments:}

Prof. Scheithauer suggested that the tumor recurred undoubtedly as a hemangioblastoma and malignant glioma might be induced with a hemangioblastoma by irradiation. 